\title{
The Variation in the Maximum Loading of a Circular Cylinder Impacted by a 2D Vortex with Time of Impact
}

\author{
Matthew N. Strasser ${ }^{\mathrm{a}, \mathrm{b}}$ \\ Email: mstrasse@uark.edu \\ Telephone: (870) 318-7278 \\ $\underline{\text { R. Panneer Selvam }}^{\mathrm{a}}$ \\ Email: rps@uark.edu \\ Telephone: (615) 212-9317
}

${ }^{a)}$ Department of Civil Engineering, University of Arkansas, Fayetteville, Arkansas, 72701, USA. Physical Address: 4190 Bell Engineering, Fayetteville, AR 72701.

${ }^{b)}$ Corresponding author.

\begin{abstract}
Direct numerical simulation is used to study the loading of a rigid, circular cylinder impacted by a $2 D$ vortex. The vortex travels within a stream of fluid characterized by Reynolds number of 150. Vortex impact occurs at twenty-five different times within one vortex shedding cycle. Substantial variation is observed in the maximum values of the drag and lift force coefficients. This variation is due to interaction between the impinging vortex and those attached to the cylinder. As the radius of the impinging vortex is increased from one to three times the cylinder's diameter, the variation in maximum force coefficients with time of impact decreases. The variation decreases because the larger vortex alters the flow field and vortex shedding cycle prior to impacting the cylinder. For structures impacted by a vortex similar in size, significant under-prediction of the maximum loading may occur if variation in loading with vortex impact time is not considered.
\end{abstract}

\section{Keywords}

- Vortex

(C) 2015. This manuscript version is made available under the Elsevier user license http://www.elsevier.com/open-access/userlicense/1.0/ 
- Vortex Loading

- Vortex-Body Interaction

- Direct Numerical Simulation

\section{Introduction}

Structural loading of a body impacted by a vortex traveling in a stream of fluid is a relevant problem in numerous fields, such as the aerospace (Tamura et al., 2008) and structural design (Rockwell, 1998) industries. Generally stated, upstream bodies shed vortices that interact with downstream bodies resulting in noise and vibration; specific examples include propellers, stabilizers, offshore platforms, and high-rise buildings. Body-vortex interaction (BVI) is classified according to the vortex orientation relative to the impacted body (A thorough review of the classifications is provided by Filippone \& Afgan (2008)). The most relevant class of interaction is "parallel", meaning the cylinder's axis and the vortex's axis of rotation are parallel (Weiland \& Vlachos, 2009).

The complexity of BVI encourages the use of numeric simulation, although the literature does report some experimental investigation (Ilie, 2009). Most numeric simulation in the literature is directed towards the aerospace industry, thus the considered Reynolds number is high $\left(\operatorname{Re}>10^{5}\right)$. In general, very little work has been performed on vortex-induced structural loading in low-Re flows (Rival et al., 2010). Furthermore, there is no investigation of the possible added complexities present when the impinging vortex impacts a body already shedding vortices. The current study is the critical first step in identifying the maximum loading that an impinging vortex of fixed maximum tangential velocity can produce on a structure as discussed in the authors' subsequent work (Strasser \& Selvam, 2015). 
In the present study, the impact of a rigid, circular cylinder by an impinging vortex is directly simulated. Vatistas' (1991) vortex profile is modified to reach a specified maximum tangential velocity for any value of exponent " $n$ " (Discussed in Eqs. (3-5)). The vortex travels within a free stream characterized by Re of 150 and impacts the cylinder at different times during one cycle of vortex shedding. Variation in the resulting maximum drag and lift force coefficients is quantified, and the underlying phenomenon responsible for the variation is discussed. The radius of the impinging vortex is incremented from one to three times the diameter of the cylinder, and the influence of impinging vortex size on the variation in maximum force coefficients with vortex impact time is assessed.

\section{Nomenclature}

A - Projected area $\left(\mathrm{m}^{2}\right)$

$\mathrm{Cd}$ - Drag force coefficient $\left(2 \cdot \mathrm{F}_{\mathrm{d}} / \rho \cdot \mathrm{U}_{\infty}{ }^{2} \cdot \mathrm{A}\right)$

$\mathrm{Cd}(+)$ - Maximum positive drag coefficient amplitude

$\mathrm{Cd}(-)$ - Maximum negative drag coefficient amplitude

$\mathrm{Cl}$ - Lift force coefficient $\left(2 \cdot \mathrm{F}_{1} / \rho \cdot \mathrm{U}_{\infty}{ }^{2} \cdot \mathrm{A}\right)$

$\mathrm{Cl}(+)$ - Maximum positive lift coefficient amplitude

$\mathrm{Cl}(-)$ - Maximum negative lift coefficient amplitude

D - Diameter of the cylinder

$F_{d}-$ Drag force

$\mathrm{F}_{1}$ - Lift force

$\mathrm{f}$ - Frequency of vortex shedding

$\mathrm{n}$ - Exponent for Vatistas' vortex model

$\mathrm{P}_{\infty}-$ Ambient/Reference Pressure

$\mathrm{P}^{*}$ - Dimensionless pressure $\left(\mathrm{P} / \rho \cdot \mathrm{U}_{\infty}{ }^{2}\right)$

$\operatorname{Re}-$ Reynolds number $\left(D \cdot U_{\infty} / v\right)$

$\mathrm{r}$ - Radial ordinate of the vortex 
$\mathrm{r}_{\mathrm{c}}-$ Critical radius for the vortex

$r_{p}$ ' - Radial distance between the vortex center and the boundary node

$\mathrm{St}$ - Strouhal number, dimensionless vortex shedding frequency $\left(\mathrm{f} \cdot \mathrm{D} / \mathrm{U}_{\infty}\right)$

$\mathrm{T}_{\text {lag }}$ - Time required for the vortex and cylinder centers to align $\left(\mathrm{X}_{\mathrm{o}} / \mathrm{U}_{\infty}\right)$

$\mathrm{T}^{*}-$ Period of vortex shedding for $\mathrm{Re}=150(1 / \mathrm{St})$

$\mathrm{t}$ - Time

$\mathrm{t}^{*}$ - Dimensionless time $\left(\mathrm{t} \cdot \mathrm{U}_{\infty} / \mathrm{D}\right)$

U - Horizontal velocity

$\mathrm{U}_{\infty}-$ Bulk velocity of the fluid stream

$\mathrm{U}^{*}$ - Dimensionless horizontal velocity $\left(\mathrm{U} / \mathrm{U}_{\infty}\right)$

$\mathrm{V}-$ Vertical velocity

$\mathrm{V}_{\theta}$ - Tangential velocity of the vortex

$\mathrm{V}^{*}$ - Dimensionless vertical velocity $\left(\mathrm{V} / \mathrm{U}_{\infty}\right)$

$\mathrm{X}$ - Horizontal ordinate

$\mathrm{X}_{\mathrm{o}}$ - Starting location of the vortex

$\mathrm{X}^{*}$ - Dimensionless horizontal ordinate $(\mathrm{X} / \mathrm{D})$

$X^{\prime}$ - Horizontal ordinate of translating reference frame attached to vortex

$\mathrm{x}_{\mathrm{p}}$ - Horizontal coordinate of boundary node with respect to cylinder center

$\mathrm{x}_{\mathrm{p}}{ }^{\prime}$ - Horizontal coordinate of boundary node with respect to vortex center

$\mathrm{Y}-$ Vertical ordinate

$\mathrm{Y}^{*}$ - Dimensionless vertical ordinate $(\mathrm{Y} / \mathrm{D})$

$Y^{\prime}$ - Vertical ordinate of translating reference frame attached to vortex

$y_{p}-$ Vertical coordinate of boundary node with respect to cylinder center

$\mathrm{y}_{\mathrm{p}}$ ' - Vertical coordinate of boundary node with respect to vortex center

$\alpha$ - Dimensionless angular velocity of the vortex

$\delta \mathrm{t}^{*}-$ Solution time step

$\Delta \mathrm{r}$ - Radial node spacing

$\rho$-Density of the fluid

$v$ - Kinematic viscosity of the fluid

BVI - Body Vortex Interaction 
CW - Clockwise

CCW - Counter Clockwise

L-O - Lamb-Oseen

NSD - Mean Normalized Standard Deviation

RCVM - Rankine Combined Vortex Model

S-K - Scully-Kaufmann

TOI - Time of Impact

UP - Under Prediction

VSC-Vortex Shedding Cycle

\section{Model and Numerical Details}

\subsection{Problem Description and Parameters}

The parallel BVI problem is illustrated in Figure 1. The impinging vortex is introduced at some point $\left(\mathrm{X}_{\mathrm{o}}\right)$ to the left of the cylinder and travels within the fluid stream at bulk velocity $\left(\mathrm{U}_{\infty}\right)$ to impact the cylinder. The term "parallel” implies that the cylinder's axis and the vortex's axis of rotation are parallel. In the present study, "direct" impact is considered, meaning these parallel axes are on the same horizontal line.

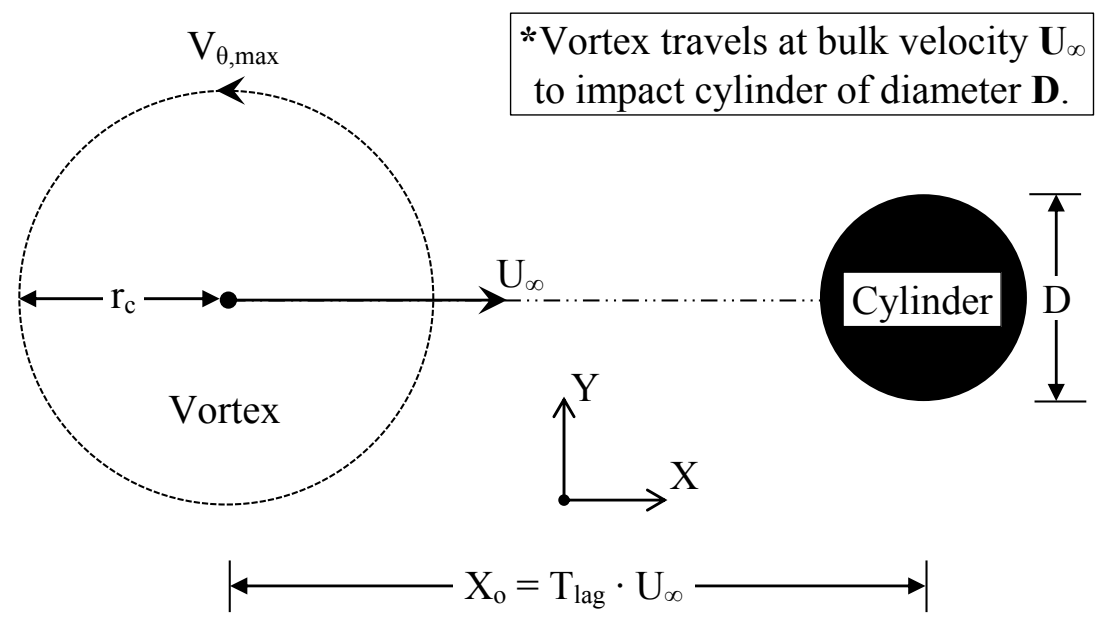

Figure 1:Schematic of the parallel BVI problem. 
The free stream Re is kept constant at 150 by fixingthe cylinder diameter, free stream bulk velocity, and kinematic viscosity at dimensionless values of $\mathrm{D}=1, \mathrm{U}_{\infty}=1$, and $v=1 / 150$ respectively. The density of the fluid is dimensionless $\rho=1$. The tangential velocity $\left(V_{\theta}\right)$ increases with radius $(r)$ from the vortex center, reaching maximum value $\left(V_{\theta \text {,max }}\right)$ at the critical radius $\left(r_{c}\right)$ and then diminishing for $r>r_{c}$. The critical radius of the impinging vortex is varied from $1 \cdot D \leq r_{c} \leq 3 \cdot D . V_{\theta, \max }$ is the product of $r_{c}$ and the dimensionless angular velocity $(\alpha)$ and is equal to unity for all simulations $\left(\mathrm{V}_{\theta, \max }=\mathrm{r}_{\mathrm{c}} \cdot \alpha=1\right)$. Note that this implies $\alpha=\mathrm{r}_{\mathrm{c}}{ }^{-1}$, meaning the rotation rate of the impinging vortex progressively slows as the vortex size increases. Drag $\left(\mathrm{F}_{\mathrm{d}}\right)$ and lift $\left(\mathrm{F}_{1}\right)$ forces on the cylinder are computed by integrating the surface pressure and shear. The forces are then re-dimensioned to the typically-discussed $\operatorname{drag}\left(\mathrm{Cd}=2 \cdot \mathrm{F}_{\mathrm{d}} / \rho \cdot \mathrm{U}_{\infty}{ }^{2} \cdot \mathrm{A}\right)$ and lift $\left(\mathrm{Cl}=2 \cdot \mathrm{F}_{1} / \rho \cdot \mathrm{U}_{\infty}{ }^{2} \cdot \mathrm{A}\right)$ force coefficients. Finally, the time lag $\left(\mathrm{T}_{\text {lag }}\right)$ is specified such that the centers of the vortex and the cylinder coincide when dimensionless time $\left(\mathrm{t}^{*}\right)$ is zero. This is illustrated in Figure 1 by fixing the starting location of the vortex $\left(\mathrm{X}_{\mathrm{o}}\right)$.

\subsection{Governing Equations}

The simulation is governed by incompressible continuity and conservation of momentum, given in tensor notation in Eqs. (1) and (2) respectively. Subscript "i" is the index, and "i and j"are 1 for " $x$ " or 2 for " $y$ ". Subscript " $t$ " corresponds to time, and a comma implies differentiation with respect to the following subscript. The governing equations are non dimensionalized with respect to $\mathrm{D}, \mathrm{U}_{\infty}, v, \rho$, and the ambient pressure $\left(\mathrm{P}_{\infty}\right)$. The accuracy of the convection approximation is enhanced using the balance tensor diffusion scheme (Dukowicz \& Ramshaw (1979) and Selvam (1998));specifically, “- $-\delta t^{*} / 2\left(U_{j}{ }^{*} U_{k}{ }^{*} U_{i, j}{ }^{*}\right), k$ " is added to the convection term $\mathrm{U}_{\mathrm{j}}{ }^{*} \mathrm{U}_{\mathrm{i}, \mathrm{j}}{ }^{*}$ where $\delta \mathrm{t}^{*}$ is the solution time step. Selvam (1997a) provides further information concerning the implementation of the balance tensor diffusion scheme. 


$$
\begin{gathered}
U_{i, i}^{*}=0 \\
U_{i, t}^{*}+U_{j}^{*} U_{i, j}^{*}+P_{, i}^{*}+\operatorname{Re}^{-1}\left[U_{i, j}^{*}+U_{j, i}^{*}\right]_{, j}=0
\end{gathered}
$$

\section{$\underline{\text { 2.2.1 Vortex Models }}$}

Detailed review of viscous vortex velocity profiles is reported in Bhagwat and Leishman (2002). The three most commonly-used profiles are Scully/Kaufmann (S-K), Lamb-Oseen (L-O), and Rankine Combined (RCVM). Selvam et al. (2002, 2003, \& 2005) use the RCVM in their previous work due to its mathematical simplicity. However, the abrupt discontinuity in the tangential velocity profile at $r=r_{c}$ is a source of substantial numerical error in transport of the impinging vortex. Alternative vortex profiles are explored to reduce numerical error and to provide more realistic representation of vortices.

Vatistas (1991) presents a robust model, defined by Eq. (3), which can approximatethe S$\mathrm{K}, \mathrm{L}-\mathrm{O}$, and RCVM profiles by setting the exponent " $\mathrm{n}$ " to equal 1, 2, and 100 respectively, as shown in Figure 2a. Vatistas' n $=2$ model accuratelyrepresents measured rotor tip vortices (Bhagwat \& Leishman, 2000), hence, it is considered for the current study.

$$
\mathrm{V}_{\theta}(\mathrm{r})=\alpha \cdot \mathrm{r}_{\mathrm{c}} \cdot\left[\frac{1}{\left(\mathrm{r} / \mathrm{r}_{\mathrm{c}}\right)^{2 \mathrm{n}}+1}\right]^{\frac{1}{\mathrm{n}}}
$$

The primary issue with Eq. (3) is that $V_{\theta, \max }$ is not equal to $\alpha \cdot r_{c}$, but rather varies when $n$ is changed ( $\mathrm{n}=1,2$, and 100 results in $\mathrm{V}_{\theta \text {,max }}$ of $0.5,0.707$, and $\approx 1.0$ respectively) as shown in Eq. (4).

$$
\mathrm{V}_{\theta, \max }=\frac{\alpha \cdot \mathrm{r}_{\mathrm{c}}}{2^{\frac{1}{\mathrm{n}}}}
$$

Eq. (3) is multiplied by $2^{1 / \mathrm{n}}$, as shown in Eq. (5), so that $\mathrm{V}_{\theta, \max }=\alpha \cdot \mathrm{r}_{\mathrm{c}}$ for all $\mathrm{n}$.

$$
V_{\theta}(r)=\alpha \cdot r \cdot\left[\frac{2}{\left(r / r_{c}\right)^{2 n}+1}\right]^{\frac{1}{n}}
$$


Comparison of $\mathrm{n}=2$ profiles defined by Eqs. (3) and (5) is given in Figure 2b. Eq. (5) is an accurate representation of the original profile given by Eq. (3), but it is scaled to the desired value of $V_{\theta, \max }=\alpha \cdot r_{c}$. The modified Vatistas' $n=2$ profilegiven by Eq. (5) is the tangential velocity profile of the vortex in the current study.

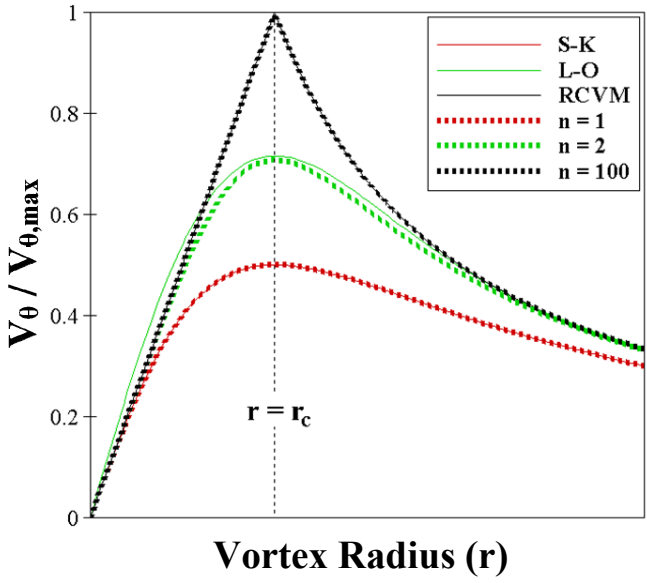

(a)

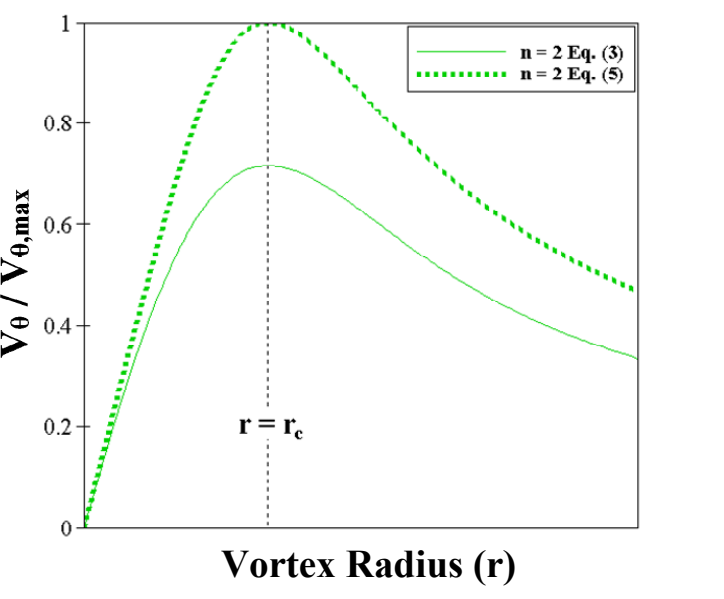

(b)

Figure 2:(a) Comparison of common viscous profiles with Eq. (3) and (b)Comparison of $\mathrm{n}=2$ profiles computed with Eq. (3) and the normalization therof given in Eq. (5).

\subsection{Numerical Method}

This work uses a finite element numeric model based on the node-centered, nonstaggered control volume model given by Selvam (1997a).The Backward Euler time advancement scheme is incorporated. Solution of the pressure and velocity field at each time step follows the four step advancement procedure outlined below, where steps 1 and 3 incorporate pre-conditioned conjugate gradient solvers.

1. Solve $\mathrm{U}_{\mathrm{i}}^{*}$ from Eq.(2).

2. Update velocity field: $\mathrm{U}_{\mathrm{i}}^{* *}=\mathrm{U}_{\mathrm{i}}^{*}+\delta \mathrm{t}^{*} \cdot \mathrm{P}_{\mathrm{i}}^{*}$

3. Solve for pressure field: $\left(\mathrm{P}_{\mathrm{i}}^{*}\right)_{\mathrm{i}}=\mathrm{U}_{\mathrm{i}, \mathrm{i}}{ }^{* *} / \delta \mathrm{t}^{*}$

4. Correct velocity for incompressibility: $\mathrm{U}_{\mathrm{i}}^{*}=\mathrm{U}_{\mathrm{i}}^{* *}-\delta \mathrm{t}^{*} \cdot \mathrm{P}_{, \mathrm{i}}^{*}$

The checkerboard pressure field is eliminated using equal-order interpolation of the pressure and velocity terms, along with step 2 . The higher order portion of the convection term and the 
diffusion term are treated implicitly, removing the time step restriction for numerical stability. A constant time step of $\delta \mathrm{t}^{*}=0.01$ is used for all simulations so that the Courant-Friedrichs-Lewy (CFL) number is always less than one. At each time step, steps 1-4 are iterated until the average residual at each node falls below $10^{-7}$. Selvam (1997b) provides further discussion of the finite element methodology and solution algorithm.

\subsection{Domain and Boundary Conditions}

A dimensioned schematic of the domain and illustration of the grid are provided in Figure 3and Figure 4 respectively. Upstream and span-wise boundaries are located $12.5 \cdot \mathrm{D}$ from the cylinder center, and the downstream boundary is $42.5 \cdot \mathrm{D}$ from the cylinder center. The surface of the cylinder is defined by 180 nodes spaced at $2^{\circ}$ increment. The first radial node spacing $\left(\Delta \mathrm{r}_{1}\right)$ from the cylinder surface is $0.0075 \cdot \mathrm{D}$, with subsequent radial spacing given by $\Delta \mathrm{r}_{\mathrm{i}}$ $=1.10 \cdot \Delta \mathrm{r}_{\mathrm{i}-1}$ up to a maximum spacing of $0.25 \cdot \mathrm{D}$. Beyond this point, constant radial node spacing of $0.25 \cdot \mathrm{D}$ is used.

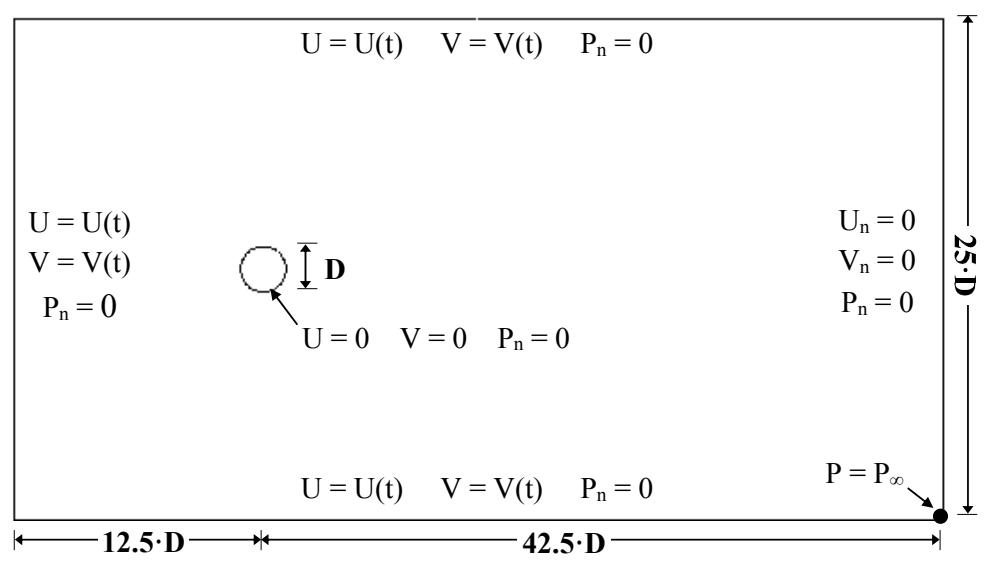

Figure 3: Illustration of the computational domain and boundary conditions. 


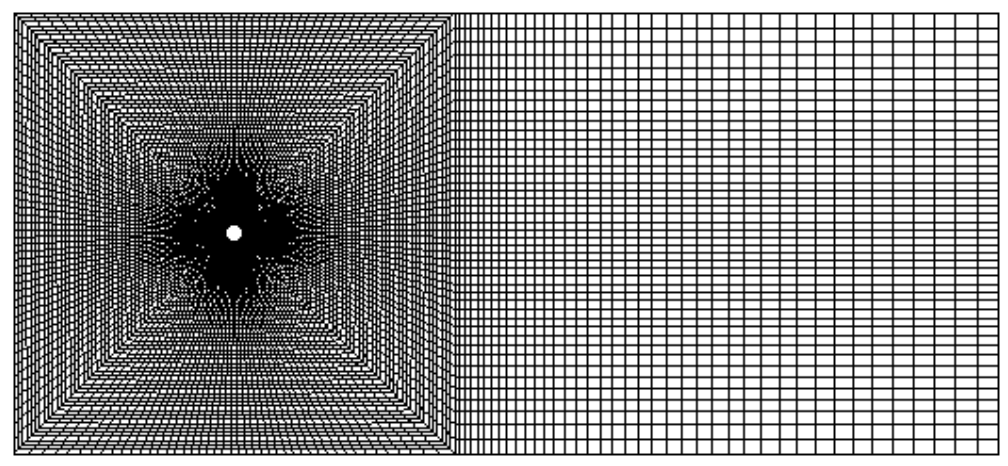

Figure 4: Illustration of the grid.

Boundary conditions are indicated in Figure 3. The no-slip condition is applied at the surface of the cylinder. The downstream boundary is located sufficiently far from the cylinder for steady flow to re-establish (note that subscript " $n$ " implies normal derivative). The pressure value in the lower downstream corner is specified to the ambient pressure, as is needed for the numerical solution. Also, the derivative of pressure normal to the domain boundaries and the cylinder's surface is equal to zero. Finally, the velocity is specified along the upstream and spanwise boundaries of the domain as a transient boundary condition computed for each boundary node at each time step. The vortex is introduced using this transient boundary condition, as first described by Selvam (1985).

The Schematic provided in Figure 5 aids in understanding the computation of the transient velocity boundary conditions. The XY coordinate system originates at the center of the cylinder and is fixed. This system relates the location of the boundary nodes, for example " $\mathrm{P}\left(\mathrm{x}_{\mathrm{p}}, \mathrm{y}_{\mathrm{p}}\right)$ ", to the center of the cylinder. The second coordinate system, $\mathrm{X}^{\prime} \mathrm{Y}$ ', is a moving reference frame that originates at the center of the vortex. The critical piece of information linking the two coordinate systems is that the vortex and cylinder centers coincide when $\mathrm{t}^{*}=0$. This means $\mathrm{t}^{*}$ is negative when the vortex is to the left of the cylinder and positive when the vortex is to the right of the cylinder. 


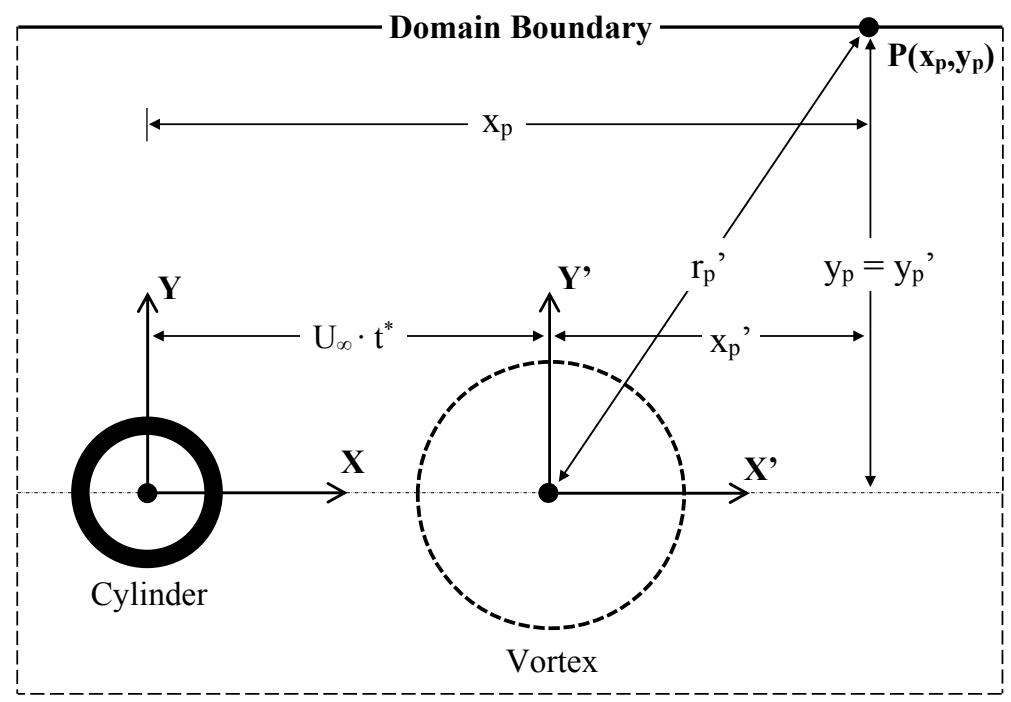

Figure 5: Schematic of the cylinder and vortex used to develop transient boundary conditions.

The coordinates of each boundary point $(P)$ remains constant $\left(x_{p}, y_{p}\right)$ within the fixed coordinate system. The location of the vortex center relative to each boundary point $\left(\mathrm{x}_{\mathrm{p}}{ }^{\prime}, \mathrm{y}_{\mathrm{p}}{ }^{\prime}\right)$ is defined at each time step via Eqs. (6a) and (6b). Subsequently, the radial distance between the vortex center and each boundary point $\left(\mathrm{r}_{\mathrm{p}}{ }^{\prime}\right)$ is computed via Eq. (7).

$$
\begin{gathered}
\mathrm{x}_{\mathrm{p}}{ }^{\prime}=\mathrm{x}_{\mathrm{p}}-\mathrm{U}_{\infty} \cdot \mathrm{t}^{*} \\
\mathrm{y}_{\mathrm{p}}{ }^{\prime}=\mathrm{y}_{\mathrm{p}} \\
\mathrm{r}_{\mathrm{p}}{ }^{\prime}=\left(\mathrm{x}_{\mathrm{p}}^{\prime 2}+\mathrm{y}_{\mathrm{p}}^{\prime 2}\right)^{1 / 2}
\end{gathered}
$$

Now, $V_{\theta}\left(r=r_{p}{ }^{\prime}\right)$ is computed using the profile defined previously in Eq. (5). Finally, Eqs. (8a) and $(8 b)$ are used to transform the tangential velocity into horizontal and vertical components and add them to the stream velocity $\mathrm{U}_{\infty}$.

$$
\begin{gathered}
\mathrm{U}(\mathrm{t})=\mathrm{U}_{\infty}-\mathrm{V}_{\theta}\left(\mathrm{r}=\mathrm{r}_{\mathrm{p}}{ }^{\prime}\right) \cdot \mathrm{y}_{\mathrm{p}}{ }^{\prime} / \mathrm{r}_{\mathrm{p}}{ }^{\prime} \\
\mathrm{V}(\mathrm{t})=\mathrm{V}_{\theta}\left(\mathrm{r}=\mathrm{r}_{\mathrm{p}}{ }^{\prime}\right) \cdot \mathrm{x}_{\mathrm{p}}{ }^{\prime} / \mathrm{r}_{\mathrm{p}}{ }^{\prime}
\end{gathered}
$$

Eqs. (8a) and (8b) are valid for any position within the domain, and they are used to initialize the velocity profile within the domain. 


\subsection{Numerical Validation}

Validating the model and grid is necessary prior to beginning the study. The accuracy with which the boundary layer is resolved is assessed by simulating steady flow over the cylinder for several values of Re commonly used in benchmark problems. Mean Cd along with the Strouhal number (St) are reported in Table 1 along with values from other computational studies from the literature. Values of $\mathrm{Cd}$ and $\mathrm{St}$ from the present study agree with the literature to within $2.25 \%$ and $1.10 \%$, which are acceptable error bounds for the current study.

Table 1: Comparison of mean $\mathrm{Cd}$ and Stfrom the current study with values from the literature.

\begin{tabular}{|c|cc|cc|cc|}
\hline \multirow{2}{*}{$\operatorname{Re}$} & \multicolumn{2}{|c|}{ (Mittal, 2005) } & \multicolumn{2}{c|}{ (Qu et al., 2013) } & \multicolumn{2}{c|}{ Current } \\
& $\mathrm{Cd}$ & $\mathrm{St}$ & $\mathrm{Cd}$ & $\mathrm{St}$ & $\mathrm{Cd}$ & $\mathrm{St}$ \\
\hline 100 & 1.322 & 0.1644 & 1.317 & 0.1649 & 1.346 & 0.1648 \\
150 & $\sim \sim \sim$ & $\sim \sim \sim$ & 1.301 & 0.1841 & 1.329 & 0.1827 \\
200 & 1.327 & 0.1947 & 1.316 & 0.1958 & 1.336 & 0.1937 \\
\hline
\end{tabular}

Nowmodel's capability to transport the impinging vortex is assessed. The tangential velocity profile of the vortex is extracted at discrete intervals as the vortex crosses the domain. The extracted profiles are then compared with the analytic velocity profile given by Eq. (5), as illustrated inFigures $\boldsymbol{6} \boldsymbol{a}$ and $\boldsymbol{6} \boldsymbol{b}$ for impinging vortex radii of $1 \cdot \mathrm{D}$ and $2 \cdot \mathrm{D}$ respectively. 


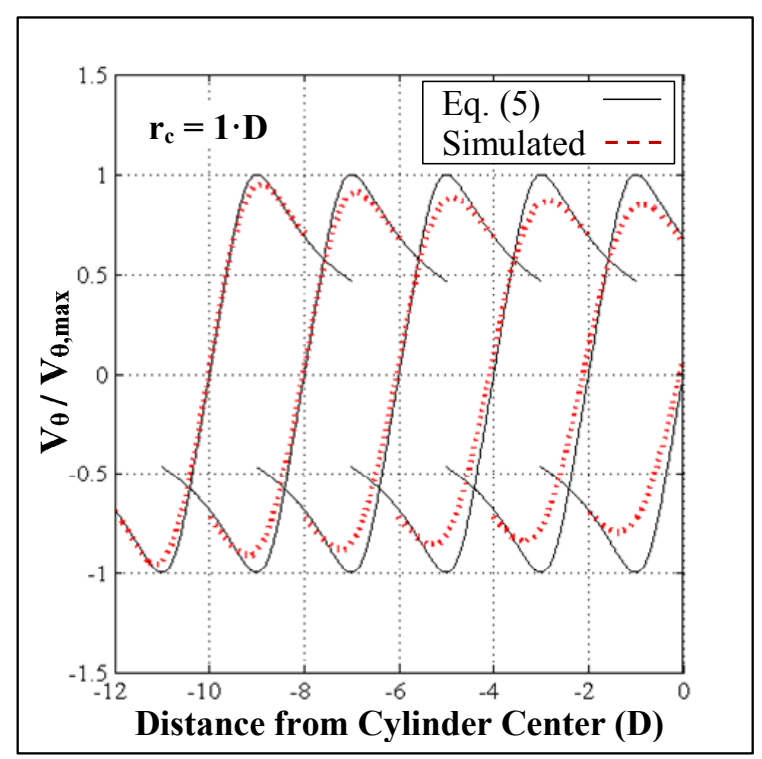

(a)

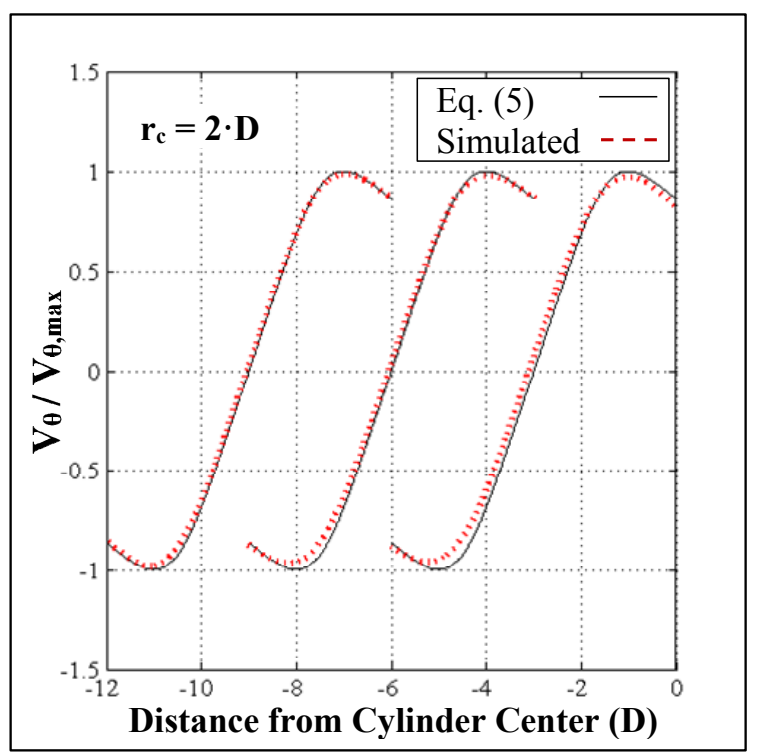

(b)

Figure 6:Comparison of simulated vortex tangential velocity profiles with analytical profiles given in Eq. (5) for impinging vortex radii (a) $1 \cdot \mathrm{D}$ and (b) $2 \cdot \mathrm{D}$.

Numerical dissipation of the $r_{c}=1 \cdot D$ vortex flattens its tangential velocity profile as shown in Figure $6 \boldsymbol{a}$ and reduces its maximum tangential velocity to $83 \%$ of of $\mathrm{V}_{\theta, \max }$ by the time it impacts the cylinder. Numerical dissipation is significant when the impinging vortex is small because few grid points are used to approximate the core of the vortex. Furthermore, $\alpha=r_{c}{ }^{-1}$ implies that the tangential velocity profile is sharper for smaller vortices, hence the tangential velocity changes rapidly between the grid points leading to further numerical error. The combination of more grid points being used to describe the vortex core and decreased sharpness of the vortex profile leads to substantial reduction in numerical dissipation of the vortex, with $97 \%$ of $V_{\theta, \max }$ being transported when $r_{c}$ is increased to $2 \cdot D$. Larger impinging vortices $\left(r_{c}=3 \cdot D\right.$ and beyond) are transported with near-100\% accuracy.Despite the numerical dissipation of the $1 \cdot \mathrm{D}$ radius vortex, the simulations still provide meaningful results and shall be considered herein. However, care must be taken when comparing results from the $1 \cdot \mathrm{D}$ radius vortex simulation with 
those from the $2 \cdot \mathrm{D}$ and $3 \cdot$ Dradius vortex simulations, as the latter two vortices are transported with greater accuracy.

\section{Approach}

Free stream flow over a cylinder at Re of 150 produces clockwise $(\mathrm{CW})$ and counter clockwise $(\mathrm{CCW})$ vortices at the top and base of the cylinder. The current study postulates that interaction between an impinging vortex and those attached to the cylinder (inter-vortex interaction) significantly influences the cylinder's loading.

The vortex shedding cycle (VSC) begins with the shedding of a CW vortex from the top of the cylinder (Figure 7a), causing peak positive amplitude of $\mathrm{Cl}$, and the midpoint is marked by the shedding of a CCW vortex from the base of the cylinder (Figure 7c), causing peak negative amplitude of $\mathrm{Cl}$ (See TOI 1 and 3 in Figure $8 \boldsymbol{b}$ ). The period of the VSC $\left(\mathrm{T}^{*}\right)$ is therefore equivalent to the period of $\mathrm{Cl}$. The relative size and strength of the $\mathrm{CW}$ and $\mathrm{CCW}$ vortices attached to the cylinder change throughout the VSC. Therefore, impact by the impinging vortex is simulated at discrete times within a single period of the VSC so that the variation in loading may be evaluated. 


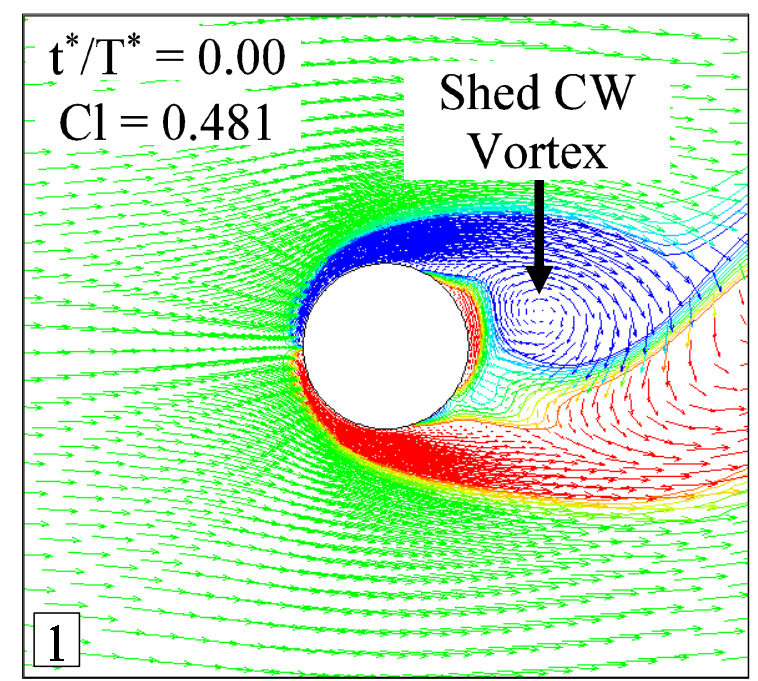

(a)

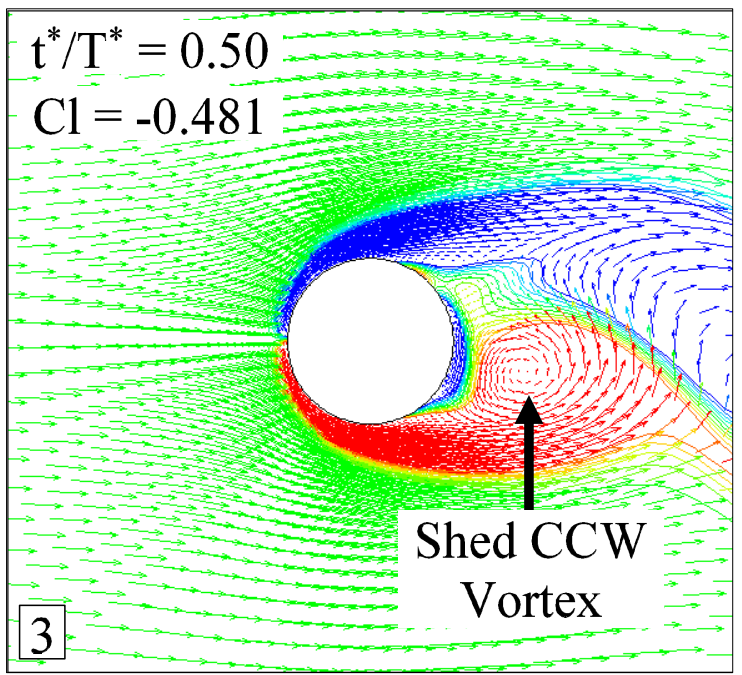

(c)

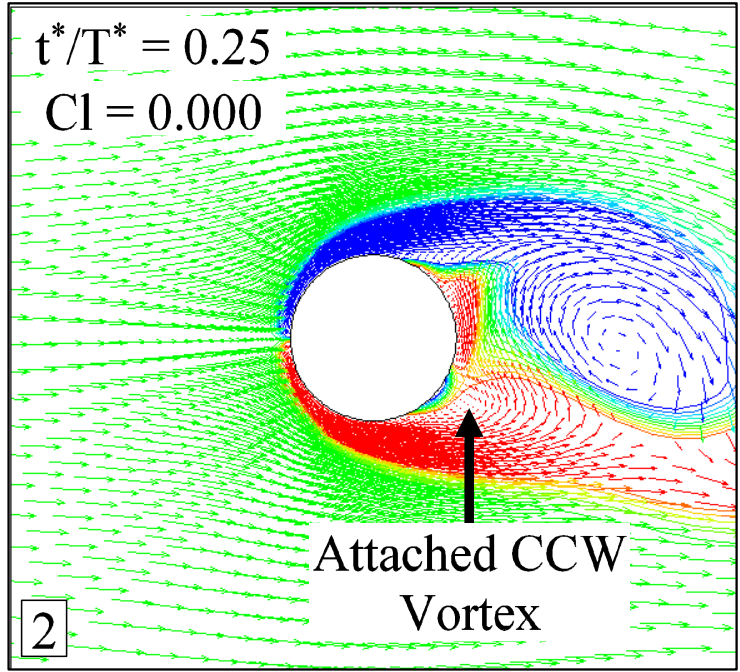

(b)

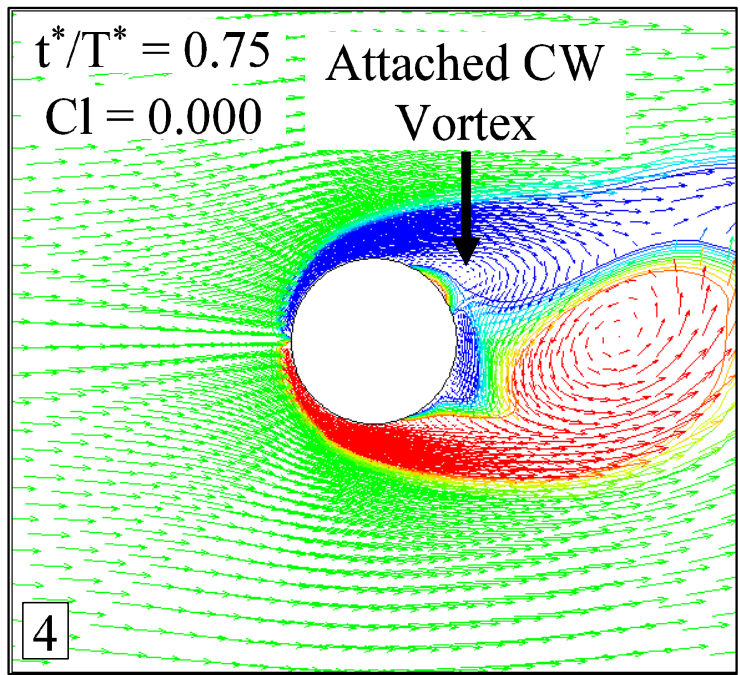

(d)

Figure 7: Vorticity contours of the shed and attached vortices in the cylinder wake at TOI 1-4 (TOI 1-4 are indicated by boxed numbers in Figure $8 \boldsymbol{b}$ ).

Free stream flow at Re of 150 is simulated, and one period of the vortex shedding cycle $\left(\mathrm{T}^{*}=5.48\right)$ is identified as shown in Figure $8 \boldsymbol{a} . \mathrm{T}^{*}$ is then discretized by assigning 25 times of vortex impact (TOI) spaced at approximate $\Delta \mathrm{t}^{*}=0.23$ interval as shown in Figure $8 \boldsymbol{b}$. Note that there is no formal, scientific basis for using $25 \mathrm{TOI}$; this high discretization resolution is simply used to ensure that variation in the maximum cylinder loading with vortex impact time is adequately captured. $\mathrm{X}_{\mathrm{o}}$ (Defined previously in Section 2.1 ) is changed so the impinging vortex 
impacts the cylinder at each TOI. In the present work, "impact" is defined as the instant the leading edges of the cylinder is touched by the impinging vortex. For each simulation, the greatest and least values of the drag and lift coefficients are extracted. These are reported as signed amplitudes $(\mathrm{Cl}(+), \mathrm{Cl}(-), \mathrm{Cd}(+)$, and $\mathrm{Cd}(-))$, computed by subtracting mean values of the drag and lift coefficients for stream flow at Re of 150 (1.329 and 0.0 respectively). This procedure is repeated for impinging vortex radii $1 \cdot \mathrm{D}, 2 \cdot \mathrm{D}$, and $3 \cdot \mathrm{D}$.

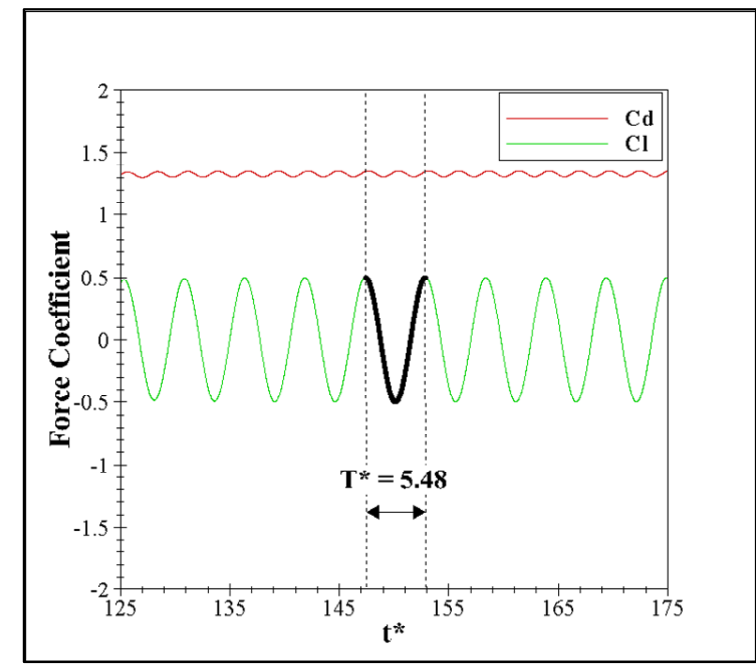

(a)

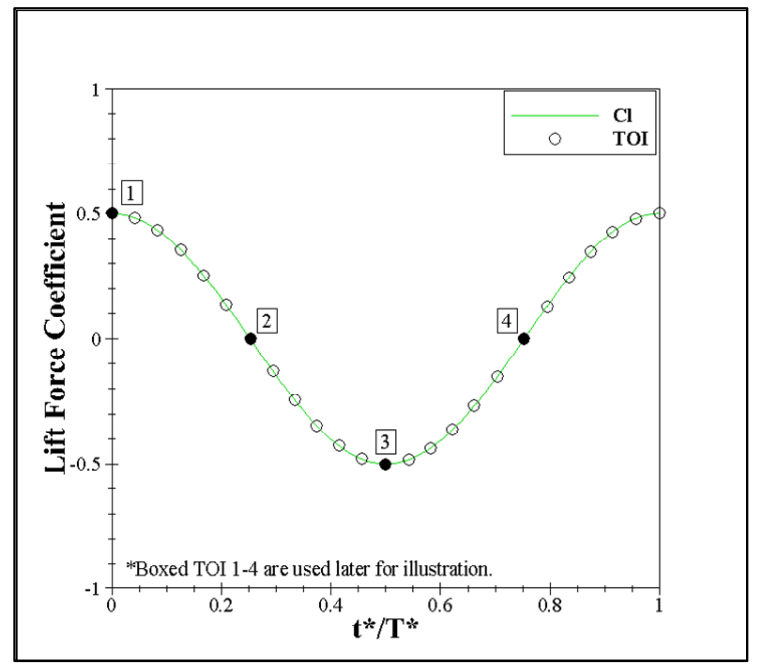

(b)

Figure 8: (a) Force coefficient time history for stream flow at $\mathrm{Re}=150$ with selected $\mathrm{T}^{*}$ indicated and (b) discretization of $\mathrm{T}^{*}$ by 25 TOI. Boxed TOI 1-4 in (b) are used for illustration later.

\section{Results and Discussion}

Maximum force coefficient amplitudes produced by impinging vortex of radius $r_{c}=1 \cdot D$ impacting the cylinder at $25 \mathrm{TOI}$ are presented. Vorticity contour plots are used to illustrate and discuss the interaction between the impinging vortex and those attached to the cylinder (intervortex interaction) that causes the variation in maximum force coefficient amplitudes with TOI. Finally, impinging vortices of radius $r_{c}=2 \cdot D$ and $3 \cdot D$ impact the cylinder at the 25 TOI and maximum force coefficient amplitudes are reported.

\subsection{Impinging Vortex: $r_{c}=1 \cdot D$}




\subsubsection{Maximum Force Coefficient Amplitudes}

Force coefficient time histories for impinging vortex impact at TOI 1-4 are provided in Figures 9a-9d. These show the large variation in both maximum force coefficient amplitudes and the caricature of the loading when the impinging vortex impacts at different times during the VSC.

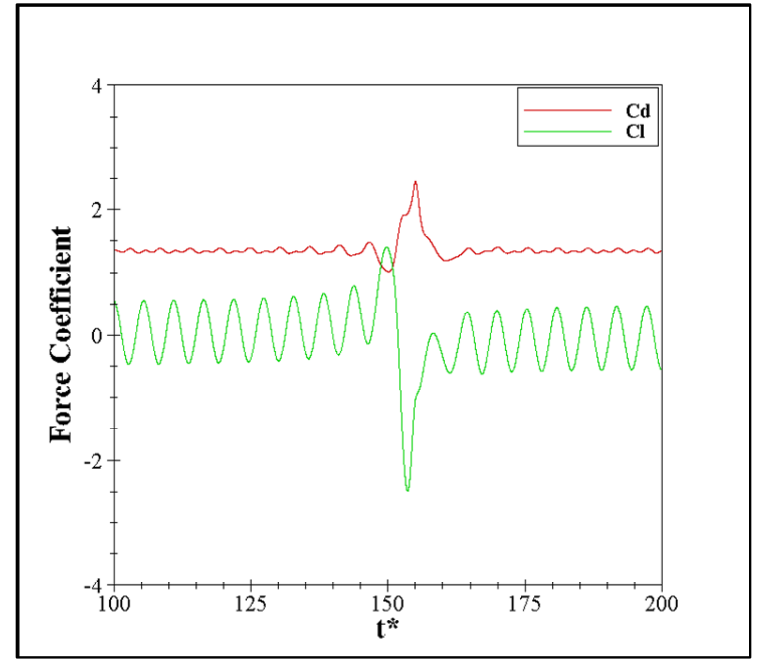

(a)

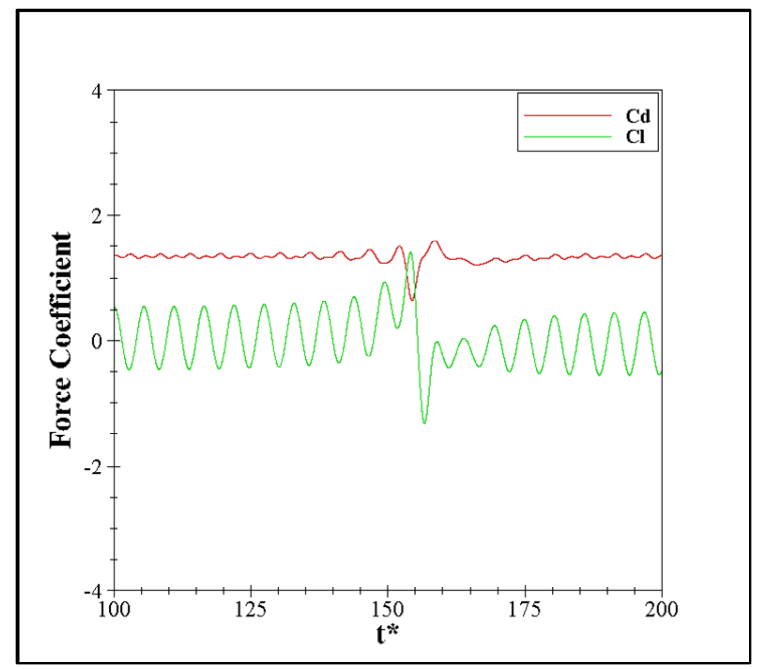

(c)

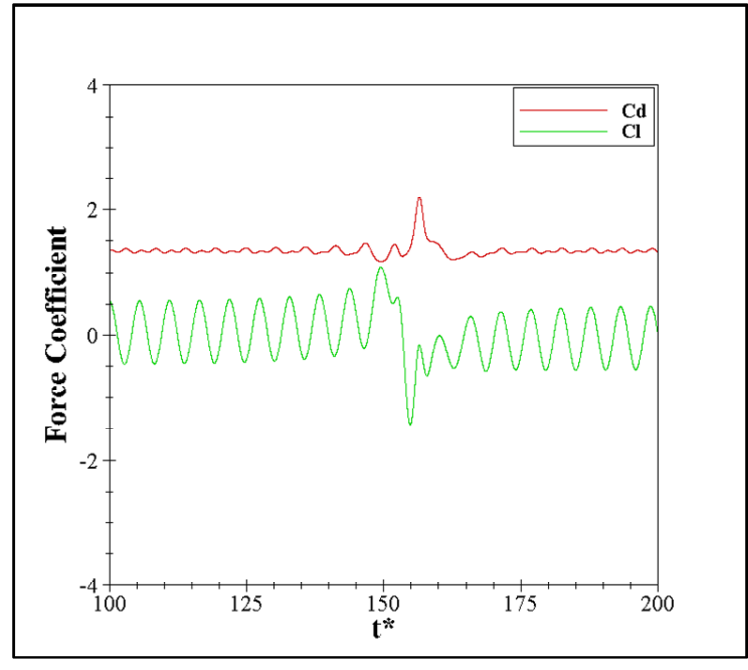

(b)

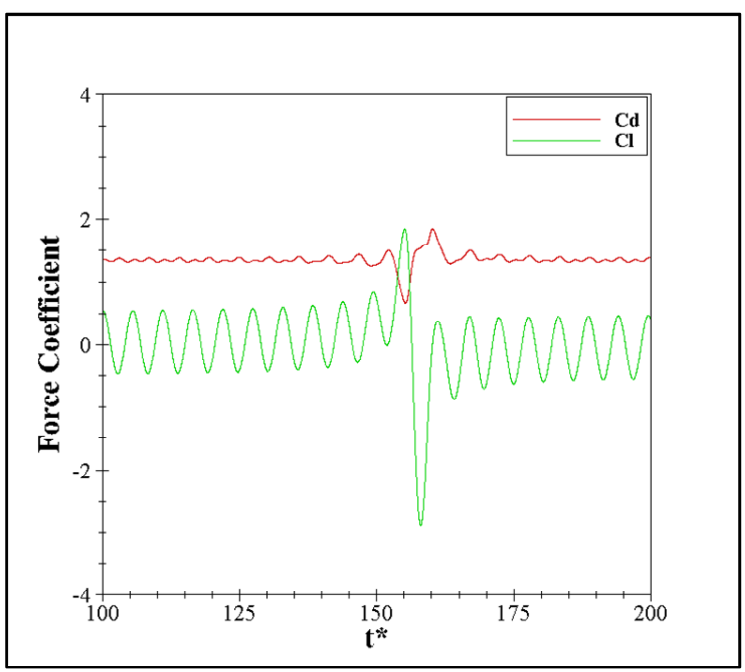

(d)

Figure 9: Force coefficient time histories for impinging 1·D vortex impact at (a) TOI 1, (b) TOI 2, (c) TOI 3, and (d) TOI 4.

The impinging vortex is now released to impact the cylinder at the 25 TOI indicated previously in Figure $8 \boldsymbol{b}$. Maximum force coefficient amplitudes are extracted from each time 
history as shown in Figure 10a and summarized in Figure 10b. The curves of maximum amplitudes are smooth and continuous. This is because the sizes of the vortices attached to the cylinder, which are believed to cause the variation in maximum force coefficient amplitudes, change continuously during vortex shedding.

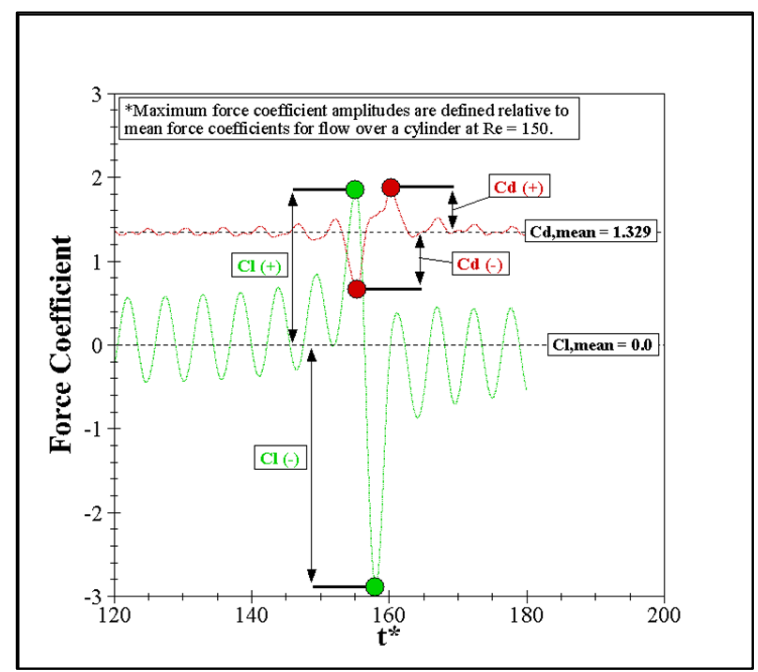

(a)

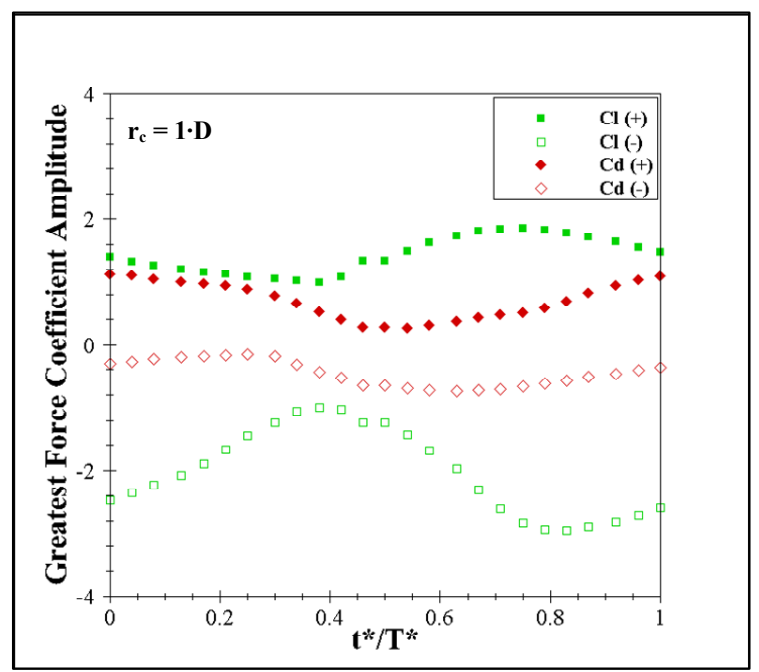

(b)

Figure 10:(a) Extraction of signed amplitudes from a typical force coefficient time history and (b) summary of signed amplitudes for impinging vortex impact at $25 \mathrm{TOI}$.

The greatest and least values from each of the four curves in Figure $\mathbf{1 0 b}$ are tabulated in Table 2 along with the under-prediction (UP) of the greatest value by the least value is also reported. Gross under-prediction of all maximum force coefficient amplitudes may occur if variation therein with TOI is not considered.

Table 2: Summary and analysis of maximum force coefficient amplitude curves for impinging vortex of radius $r_{c}=1 \cdot D$ impacting the cylinder at 25 TOI (Illustrated in Figure $8 \boldsymbol{b}$ ).

\begin{tabular}{|c|cccc|}
\hline \multirow{2}{*}{} & \multicolumn{4}{|c|}{ Maximum Force Coefficient Amplitudes } \\
\cline { 2 - 5 } & $\mathrm{Cl} \mathrm{(+)}$ & $\mathrm{Cl}(-)$ & $\mathrm{Cd} \mathrm{(+)}$ & $\mathrm{Cd} \mathrm{(-)}$ \\
\hline Greatest & 1.851 & -2.952 & 1.128 & -0.735 \\
Least & 1.000 & -0.994 & 0.275 & -0.145 \\
UP & $84.99 \%$ & $197.04 \%$ & $310.42 \%$ & $407.15 \%$ \\
\hline
\end{tabular}

*UP is under prediction. 


\subsubsection{Visualization of the Interaction between Impinging and Attached Vortices}

Vortex impact at TOI 1-4 is used to illustrate inter-vortex interaction. TOI 1-4 are

selected because the structure of the vortices attached to the cylinder at these times, due to free stream flow at $\operatorname{Re}=150$, is well-understood and bulleted below. Corresponding vorticity contours are illustrated in column "a" of Figure 11.

- $\quad$ TOI $1-\mathrm{CW}$ vortex is just shed.

- TOI $2-\mathrm{T}^{*} / 4$ from the shedding of a $\mathrm{CCW}$ vortex.

- TOI $3-\mathrm{CCW}$ vortex is just shed.

- TOI $4-\mathrm{T}^{*} / 4$ from the shedding of a $\mathrm{CW}$ vortex.
(CW - Largest, CCW - Small).

(CW - Smallest, CCW - Large).

(CW - Small, CCW - Largest).

(CW - Large, CCW - Smallest).

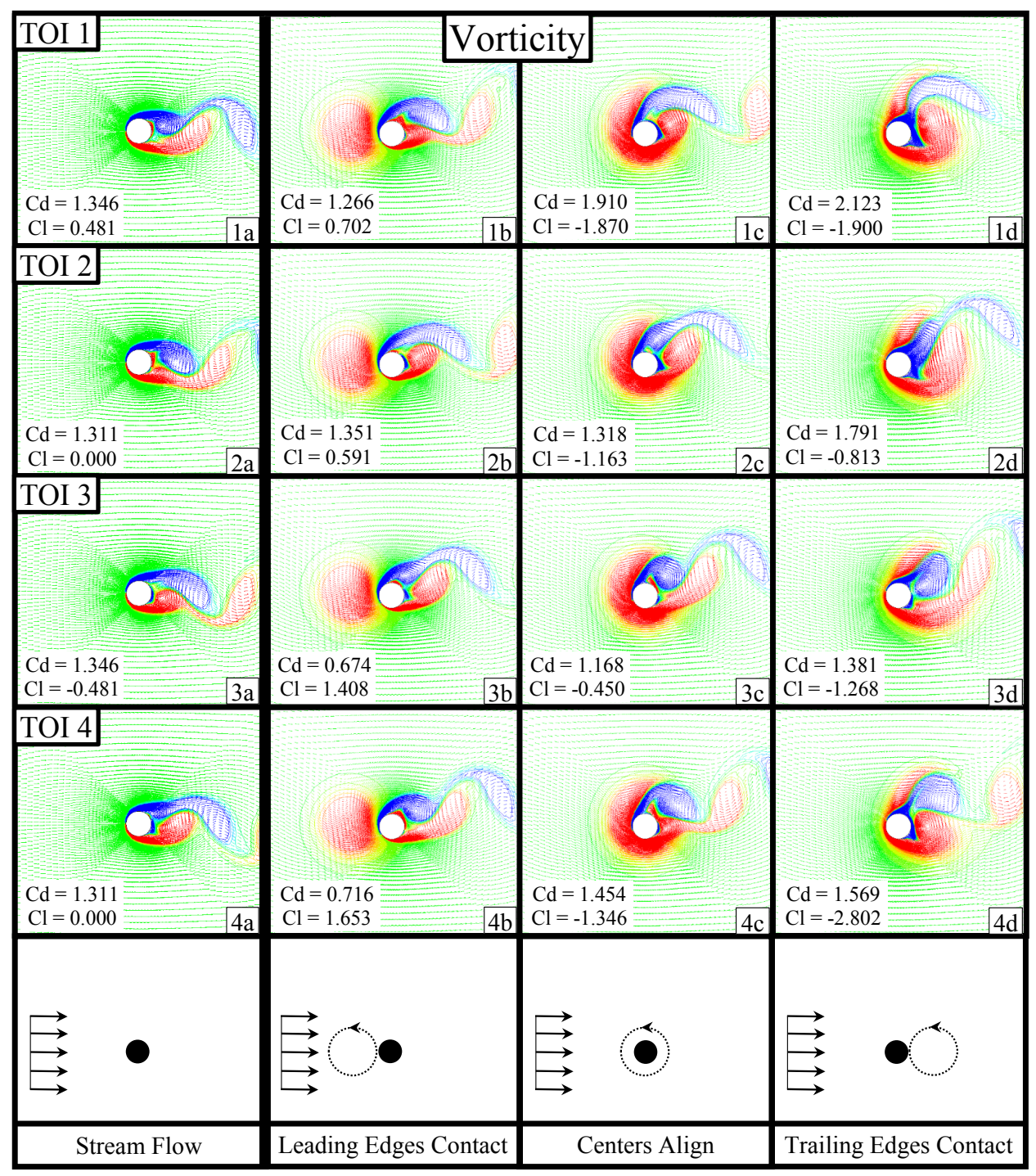


Figure 11: Vorticity contours and velocity vectors at TOI 1-4 (by row) for free stream flow over the cylinder (column a) and for the three relative positions of the vortex to the cylinder illustrated in row 5 (columns b-d).

Figure 11 illustrates the vorticity field surrounding the cylinder for impinging vortex impact at TOI 1-4. Rows 1-4 correspond to TOI 1-4, and columns b-d correspond to the three "expected" relative positions of the vortex to the cylinder illustrated in Row 5. The illustrations are of "expected" relative positions, because it is assumed that the impinging vortex continues to translate at $\mathrm{U}_{\infty}$ throughout interaction with the cylinder. Instantaneous values of $\mathrm{Cd}$ and $\mathrm{Cl}$ are provided in the lower-left corner of each figure. The visualization in Figure 11 is used to illustrate and discuss two significant forms of inter-vortex interaction. Specifically, the impinging vortex alters the positions of the vortices attached to the cylinder, and the impinging vortex delays the vortex shedding cycle as discussed subsequently in Section 4.1.2.1.

\subsubsection{Physical Interaction between Impinging and Attached Vortices}

A free stream flowing over a cylinder produces $\mathrm{CW}$ and $\mathrm{CCW}$ vortices on the cylinder surface located at "+" and “-” $90^{\circ}$ (respectively) from the free stream’s direction. For a horizontal free stream, the vortices form at the top and base of the cylinder and stretch horizontally behind the cylinder as shown in column "a" of Figure 11. As the impinging CCW vortex approaches the cylinder, its tangential velocity adds a positive, vertical velocity component to the stream over the cylinder, hence the positions of the attached vortices rotate $\mathrm{CCW}$ around the cylinder surface as shown in column "b" of Figure 11. The attached CW and CCW vortices now partially occupy the upstream and downstream edges of the cylinder respectively. The net strength of these attached vortices controls the amplitude of $\mathrm{Cd}$, as shall be discussed shortly.

Column "a" of Figure 11 illustrates the attached vortex structure at TOI 1-4 due to the free stream, while column " $b$ " illustrates the attached vortex structure when the impinging vortex impacts the leading edge of the cylinder at TOI 1-4. Comparing the attached vortex structure in 
Figures 11.1a and 11.1b, one can see that in addition to orientation, the attached vortices differ substantially in appearance. This implies that in addition to shifting the orientation of the attached vortices, the impinging vortex's tangential velocity also alters the development of the attached vortices.

The attached vortices illustrated in Figures $11.1 \mathrm{a}$ and $\mathbf{1 1 . 4 b}$ are notably similar. In fact, the attached vortex structure in each of the column " $b$ " contours resembles the attached vortex structure in the subsequent row of each column "a" contours. Specifically, Figures 11.2a and 11.1b, Figures $11.3 a$ and $11.2 b$, and Figures $11.4 a$ and $11.3 b$ exhibit similar attached vortex structure. This implies that the transient contribution of the impinging vortex's tangential velocity to the stream over the cylinder delays the free stream vortex shedding cycle by approximately $\mathrm{T}^{*} / 4$ prior to the vortex impacting the cylinder. Therefore, the actual structure of the attached vortices, when the impinging vortex is released to impact the cylinder at TOI 1-4 defined with respect to the free stream vortex shedding cycle, is:

- Impinging vortex impacts at TOI $1-\mathrm{T}^{*} / 4$ from the shedding of a $\mathrm{CCW}$ vortex.

- Impinging vortex impacts at TOI $2-\mathrm{CCW}$ vortex is just shed.

- Impinging vortex impacts at TOI $3-\mathrm{T}^{*} / 4$ from the shedding of a $\mathrm{CW}$ vortex.

- Impinging vortex impacts at TOI $4-\mathrm{CW}$ vortex is just shed.

The attached vortices illustrated in column " $b$ " are visibly larger than those in column "a". It is postulated that the delay of the vortex shedding cycle and the increased stream velocity over the cylinder (due to the impinging vortex's tangential velocity) cause the attached vortices to grow larger, thus they exert greater force on the cylinder.

\subsubsection{Loading due to Interaction between Impinging and Attached Vortices}

Discussion shall focus on inter-vortex interaction producing large force coefficient amplitudes. Interaction scenarios producing small amplitudes can be inferred but are omitted to avoid redundancy.It is convenient to begin discussion with $\mathrm{Cd}$. A vortex is a low pressure region, 
hence, the attached vortices pull the cylinder towards themselves. The amplitude of the force on the cylinder is the net force produced by the two vortices, and the sign of the amplitude is in the direction of the larger attached vortex.

The attached $\mathrm{CW}$ and $\mathrm{CCW}$ vortices are rotated to partially occupy the upstream and downstream surfaces of the cylinder, respectively, as the impinging vortex approaches. One of the attached vortices is always larger than the other. A larger $\mathrm{CW}$ vortex will result in high $\mathrm{Cd}(-)$, while a larger $\mathrm{CCW}$ vortex results in high $\mathrm{Cd}(+)$. Time histories in Figure 9 show that vortex impact at TOI 1 and 2 produce large $\mathrm{Cd}(+)$ while impact at TOI 3 and 4 produce large $\mathrm{Cd}(-)$. It is now known that the VSC is delayed $\mathrm{T}^{*} / 4$ by the impinging vortex. Therefore, the structures of attached vortices (defined in the second set of bullet points) that produce large $\mathrm{Cd}(+)$ and $\mathrm{Cd}(-)$ are as expected.

The largest values of $\mathrm{Cl}(+)$ and $\mathrm{Cl}(-)$ occur when the impinging vortex impacts at TOI 4 (Figure 9d) as a $\mathrm{CW}$ vortex is being shed. The occurrence of the large value of $\mathrm{Cl}(+)$ is expected, as the attached $\mathrm{CW}$ vortex is at its largest size, and therefore, exerts maximum pull along the top surface of the cylinder. In addition to exerting this pull, the large CW vortex shifts the path of the impinging vortex. In the absence of attached vortices, the impinging vortex will engulf the cylinder, simultaneously producing similar pull along the top and base of the cylinder. However, the large $\mathrm{CW}$ vortex that is now shifted towards the upstream edge of the cylinder interacts with and resists the impinging vortex. The path of the impinging vortex is shifted downward, and it merges with the attached CCW vortex attached to the base of the cylinder. The merged and now-large $\mathrm{CCW}$ vortex exerts a strong downward pull along the base of the cylinder, causing the large $\mathrm{Cl}(-)$.

\subsubsection{Summary}


The inter-vortex interaction responsible for the variation in the maximum force coefficient amplitude with vortex TOI is now understood. The VSC is delayed by $\mathrm{T}^{*} / 4$ by impinging vortex of radius $1 \cdot \mathrm{D}$, causing the attached vortices to enlarge. $\mathrm{Cd}(+)$ and $\mathrm{Cd}(-)$ are large if the attached $\mathrm{CW}$ and $\mathrm{CCW}$ vortices (respectively) are large when the impinging vortex impacts. $\mathrm{Cl}(+)$ and $\mathrm{Cl}(-)$ are both large if the attached $\mathrm{CW}$ vortex is strong when the impinging vortex impacts.

This discussion of inter-vortex interaction (Section 4.1.2) is only directly applicable to the system having parameters defined in Section 2.1. Any number of parameters may influence the interaction: Reynolds number, impinging vortex size, maximum tangential velocity of the impinging vortex relative to bulk velocity, etc. and have not yet been investigated. However, using the procedure and methodology adopted here, the inter-vortex interaction can be studied for any system.

\subsection{Impinging Vortex: $r_{c}=2 \cdot D$ and $3 \cdot D$}

The procedure presented in Section 4.1.1 is repeated to construct the curves of maximum force coefficient amplitudes for impinging vortices of radii $2 \cdot \mathrm{D}$ and $3 \cdot \mathrm{D}$ illustrated in Figures $12 a$ and $12 b$. As the size of the impinging vortex is increased, it can be seen that the curves (Figure 10b and Figures $12 a$ and 12b) of maximum force coefficient amplitude flatten as $\mathrm{r}_{\mathrm{c}}$ increases from $1 \cdot \mathrm{D}$ to $3 \cdot \mathrm{D}$. The flattening of the maximum force coefficient amplitude curves means there is less variation in loading with TOI. The impinging vortex exerts greater influence on the stream over the cylinder as its size is increased. As $r_{c}$ becomes large, the VSC is not just delayed, but rater is controlled by the impinging vortex. Consequently, TOI becomes irrelevant, as the position of the impinging vortex will dictate the structure of the vortices attached to the 
cylinder. Subsequent increase in $r_{c}$ will eventually lead to constant maximum force coefficient amplitudes for impinging vortex impact at any time.

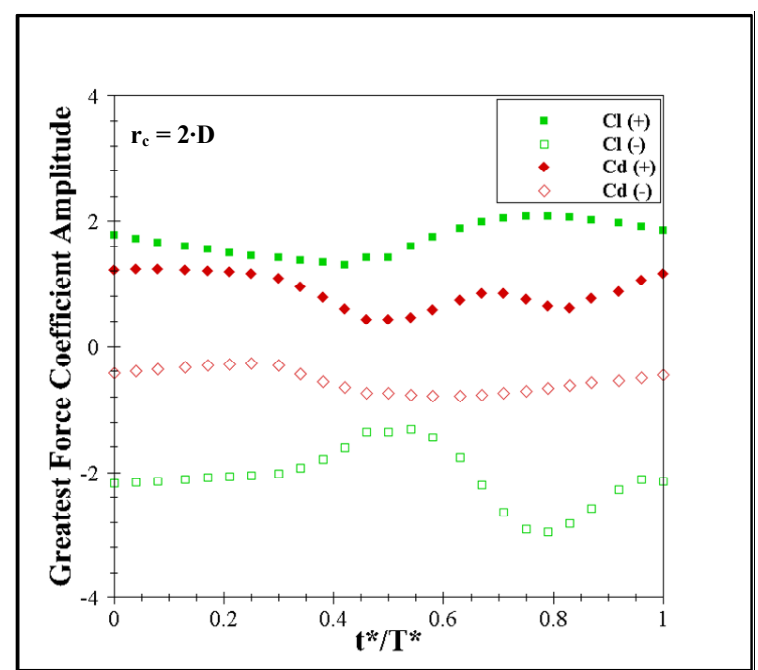

(a)

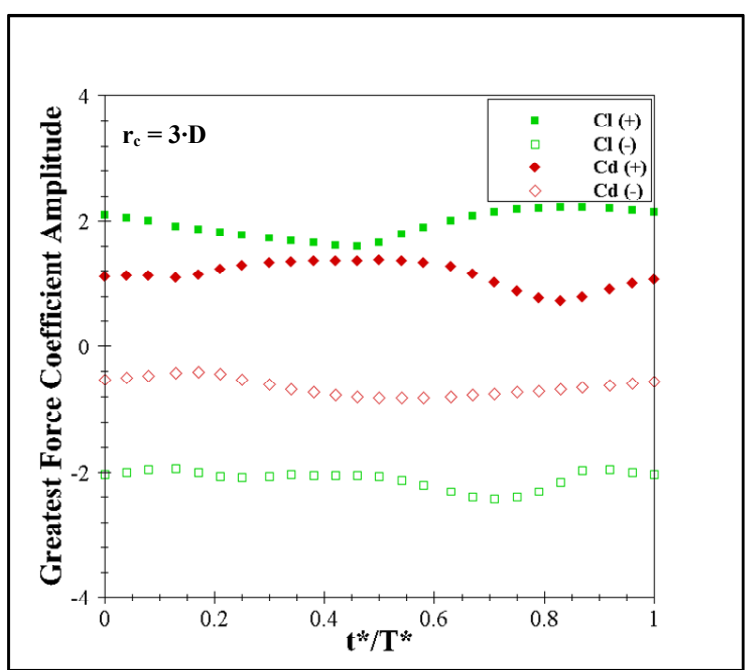

(b)

Figure 12: Maximum force coefficient amplitudes for impinging vortex impact at $25 \mathrm{TOI}$ for vortex of radius (a) $2 \cdot \mathrm{D}$ and (b) $3 \cdot \mathrm{D}$.

Mean values are computed for each maximum force coefficient amplitude curve (Figure $10 b$ and Figures $12 a$ and $12 b$ ) and reported in Table 3 along with the mean-normalized standard deviation (NSD) for each curve. Mean values of all amplitudes increase consistently as the size of the impinging vortex is increased. This is because more of the cylinder is exposed to high tangential velocity from the vortex. Also, NSD in all amplitude curves consistently decreases as the size of the impinging vortex is increased, corroborating the previous discussion.

Table 3: Mean and NSD for curves of maximum force coefficient amplitude for impinging vortex having radii of $1 \cdot \mathrm{D}, 2 \cdot \mathrm{D}$, and $3 \cdot \mathrm{D}$.

\begin{tabular}{|c|cccc|}
\hline \multirow{2}{*}{ Vortex } & \multicolumn{4}{|c|}{ Signed Maximum Amplitudes } \\
\cline { 2 - 5 } Radius & $\mathrm{4}(+)$ & $\mathrm{Cl}(-)$ & $\mathrm{Cd}(+)$ & $\mathrm{Cd}(-)$ \\
\hline 1.D & 1.432 & -2.030 & 0.707 & -0.459 \\
$2 \cdot \mathrm{D}$ & 1.710 & -2.083 & 0.886 & -0.548 \\
3.D & 1.949 & -2.109 & 1.150 & -0.647
\end{tabular}




\begin{tabular}{|l|cccc|}
\hline & \multicolumn{4}{|c|}{ Mean-Normalized St. Deviation } \\
\hline & $20.34 \%$ & $33.44 \%$ & $42.75 \%$ & $45.90 \%$ \\
& $15.62 \%$ & $22.12 \%$ & $31.30 \%$ & $32.96 \%$ \\
& $11.09 \%$ & $6.91 \%$ & $17.86 \%$ & $20.91 \%$ \\
\hline
\end{tabular}

\section{Summary and Conclusions}

The impact of an impinging vortex with a rigid circular cylinder immersed in a fluid stream is studied via 2D direct simulation. The Reynolds number based upon the free stream and the cylinder diameter (D) is 150 . The radius of the impinging vortex is varied from $1 \cdot \mathrm{D}$ to $3 \cdot \mathrm{D}$, and the maximum tangential velocity of the vortex is the same as the free stream flow. The tangential velocity profile of the impinging vortex is a normalized version of Vatistas' $n=2$ model, which gives realistic approximation of viscous vortices. The impinging vortex impacts the cylinder at 25 different times of impact (TOI) during one period of the vortex shedding cycle. For each TOI, the greatest signed amplitudes $(\mathrm{Cd}(+), \mathrm{Cd}(-), \mathrm{Cl}(+)$, and $\mathrm{Cl}(-))$ of the drag and lift coefficient are extracted. The signed amplitudes vary substantially with TOI, and trends in the signed amplitude with increasing size of the impinging vortex are documented. Based upon the content presented herein, the conclusions outlined below have been reached.

1. Maximum force coefficient amplitudes vary substantially when impinging vortex of radius 1.D impacts the cylinder at different times during the vortex shedding cycle.

a. Maximum force coefficient amplitudes range from:

i. $\mathrm{Cl}(+): 1.000$ to 1.851

ii. $\mathrm{Cl}(-)$ : -0.994 to -2.952

iii. $\mathrm{Cd}(+): 0.275$ to 1.128

iv. $\mathrm{Cd}(-)$ : -0.145 to -0.735

b. Maximum force coefficient amplitudes may be grossly under-predicted if variation with TOI is not considered.

2. Variation in maximum force coefficient amplitude with TOI is caused by interaction between the impinging vortex and the vortices attached to the cylinder.

a. As the impinging counter-clockwise $(\mathrm{CCW})$ vortex approaches the cylinder, itstangential velocity influences the stream over the cylinder.

i. The clockwise $(\mathrm{CW})$ and $\mathrm{CCW}$ vortices, originally along the top and base of the cylinder, rotate $\mathrm{CCW}$ around the cylinder. 
ii. The impinging vortex delays the shedding of the attached vortices, causing them to grow larger and exert increased loading on the cylinder.

b. If the attached $\mathrm{CW}$ vortex is large when the impinging vortex impacts: $\mathrm{Cd}(-)$, $\mathrm{Cl}(+), \& \mathrm{Cl}(-)$ will be large.

c. If the attached $\mathrm{CCW}$ vortex is large when the impinging vortex impacts: $\mathrm{Cd}(+)$ will be large.

3. Variation in maximum force coefficient amplitude with TOI decreases as the vortex size increases.

a. Curves for $\mathrm{Cd}(+), \mathrm{Cd}(-), \mathrm{Cl}(+)$, and $\mathrm{Cl}(-)$ are constructed for $25 \mathrm{TOI}$, and meannormalized standard deviation (NSD) are computed for each.

i. The greatest NSD for each impinging vortex size are:

1. $45.90 \%(1 \cdot \mathrm{D})$

2. $32.96 \%(2 \cdot D)$

3. $20.91 \%(3 \cdot \mathrm{D})$

b. NSD decreases with increasing impinging vortex size because the impinging vortex exerts increased influence on the vortex shedding cycle.

c. Continued increase in vortex size should eventually lead to no variation in maximum force coefficient amplitude with TOI.

\section{Future Work}

The current study serves as the critical first step in evaluating the maximum loading that a vortex, having fixed maximum tangential velocity, can exert on a circular cylinder. Variation in maximum force coefficient amplitude with time of impact diminishes as $r_{c}$ is increased from $1 \cdot D$ to $3 \cdot \mathrm{D}$; however, substantial variation remains present even for $r_{c}$ of $3 \cdot D$.

The next phase of the authors' work is to increase the radius of the impinging vortex further and identify the impinging vortex size where TOI ceases to influence the maximum force coefficient amplitudes. Subsequent work will focus on determining the maximum force coefficients that a vortex of fixed maximum tangential velocity can produce on a cylindrical structure. These topics are evaluated in Strasser \& Selvam (2015).

Additional topics that will be investigated include evaluation of variation in loading with time of impact when the impinging vortex size is $r_{c}<1 \cdot D$. For such small vortices, accurate transport of the impinging vortex becomes challenging, and much greater grid refinement will be 
needed. The current investigation procedure (25 TOI per $r_{c}$ value) is already computationally expensive, as individual simulations take approximately 11 hours to run for $200 \mathrm{t}^{*}$. Further grid refinement will greatly increase the computational expense, hence will likely be delayed at the present time.

\section{Acknowledgments}

This authors would like to acknowledge the National Science Foundation, the James T. Womble Endowment, and the Schueck Family Fund for providing the financial resources under which this research was conducted at the University of Arkansas. The opinions expressed herein are solely those of the authors and do not reflect those of the funding parties.

The authors would also like to express thanks to the editor and the reviewers for their thorough critique of this work, which has resulted in significant improvement and clarification of the study.

\section{References}

Bhagwat, M.J., \& Leishman, J.G., 2000. Measurements of bound and Wake Circulation on a Helicopter Rotor, J. Aircraft 37.2, 227-234.

Bhagwat, M.J., \& Leishman, J.G., 2002. Generalized Viscous Vortex model for Application to Free-Vortex Wake and Aeroacoustic Calculations, Annual Forum Proceedings-American Helicopter Society 58.2, 2042-2057.

Dukowicz, J., \& Ramshaw, J., 1979. Tensor viscosity method for convection in numerical fluid dynamics, J. Computational Physics 32.1, 71-79

Filippone, A. \& Afgan, I., 2008. Orthogonal Blade-Vortex Interaction on a Helicopter Tail Rotor, AIAA 46, 1476-1488.

Ilie, M., 2009. Numerical study of helicopter blade-vortex mechanism of interaction using largeeddy simulation, Computers and Structures 87, 758-768.

Mittal, S., 2005. Excitation of shear layer instability in flow past a cylinder at low Reynolds number, Int. J. Numer. Meth. Fluids 49, 1147-1167.

Qu, L., Norberg, C., \& Davidson, L., 2013. Quantitative numerical analysis of flow past a circular cylinder at Reynolds number between 50 and 200, J. Fluids and Structures 39, 347-370. 
Rival, D., Manejev, R., \& Tropea, C., 2010. Measurement of parallel blade-vortex interaction at low Reynolds numbers, AIAA 49, 89-99.

Rockwell, D., 1998. Vortex-Body Interactions, Annual Rev. Fluid Mech. 30, 199-229.

Selvam, R.P., 1985. Application of the Boundary Element Method for Tornado Forces on Building, Ph.D. Dissertation, Texas Tech, Lubbock, TX.

Selvam, R.P., 1997a. Computation of pressures on Texas Tech University building using large eddy simulation, J. Wind Eng. Ind. Aerodyn. 67\&68, 647-657.

Selvam, R.P., 1997b. Finite element modeling of flow around a circular cylinder using LES, J. Wind Engineering 67\&68, 129-139.

Selvam, R.P., Tarini, M.J., \& Larsen, A., 1998. Computer modelling of flow around bridges using LES and FEM, J. Wind Engineering and Industrial Aerodynamics 77\&78, 643-651.

Selvam, R. P., Roy, U.K., Jung, Y., \& Mehta, K.C., 2002. Investigation of tornado forces on 2D cylinder using computer modeling, Wind Engineering, Proc. NCWE, 342-353.

Selvam, R.P., \& Millet, P.C., 2003. Computer modeling of tornado forces on buildings, Wind and Structures 6.3, 209-220.

Selvam, R.P., \& Millet, P.C., 2005. Large eddy simulation of tornado-structure interaction to determine structural loading, Wind and Structures, 8.1, 49-60.

Strasser, M.N., \& Selvam, R.P., 2015. On the Interaction of a 2D Vortex with a Cylinder: The Influence of Vortex Size and Path on Cylinder Loading. (In review withJ. Theoretical and Computational Fluid Dynamics).

Tamura, A., Tsutahara, M., Kataoka, T., Aoyama, T., \& Yang, C., 2008. Numerical Simulation of Two-Dimensional Blade-Vortex Interactions Using Finite Difference Lattice Boltzmann Method, AIAA 46, 2235-2247.

Vatistas, G.H., Kozel, V., \& Mih, W.C., 1991. A Simpler Model for Concentrated Vortices, Experiments in Fluids 11, 73-76.

Weiland, C. \& Vlachos, P.P., 2009. A mechanism for mitigation of blade-vortex interaction using leading edge blowing flow control," Exp. Fluids 47, 411-426.

\section{Figure Captions}

Figure 1:Schematic of the parallel BVI problem.

Figure 2:(a) Comparison of common viscous profiles with Eq. (3) and (b)Comparison of $\mathrm{n}=2$ profiles computed with Eq. (3) and the normalization therof given in Eq. (6). 
Figure 3: Illustration of the computational domain and boundary conditions.

Figure 4: Illustration of the grid.

Figure 5: Schematic of the cylinder and vortex used to develop transient boundary conditions.

Figure 6:Comparison of simulated vortex tangential velocity profiles with analytical profiles given in Eq. (4) for impinging vortex radii (a) $1 \cdot \mathrm{D}$ and (b) $2 \cdot \mathrm{D}$.

Figure 7: Vorticity contours of the shed and attached vortices in the cylinder wake at TOI 1-4 (TOI 1-4 are indicated by boxed numbers in Figure $8 \boldsymbol{b}$ ).

Figure 8: (a) Force coefficient time history for stream flow at $\mathrm{Re}=150$ with selected $\mathrm{T}^{*}$ indicated and (b) discretization of $\mathrm{T}^{*}$ by 25 TOI. Boxed TOI 1-4 in (b) are used for illustration later.

Figure 9: Force coefficient time histories for impinging 1·D vortex impact at (a) TOI 1, (b) TOI 2, (c) TOI 3, and (d) TOI 4.

Figure 10: (a) Extraction of signed amplitudes from a typical force coefficient time history and (b) summary of signed amplitudes for impinging vortex impact at 25 TOI.

Figure 11: Vorticity contours and velocity vectors at TOI 1-4 (by row) for free stream flow over the cylinder (column a) and for the three relative positions of the vortex to the cylinder illustrated in row 5 (columns b-d).

Figure 12: Maximum force coefficient amplitudes for impinging vortex impact at 25 TOI for vortex of radius (a) $2 \cdot \mathrm{D}$ and (b) $3 \cdot \mathrm{D}$.

Tables

Table 1: Comparison of mean $\mathrm{Cd}$ and Stfrom the current study with values from the literature.

\begin{tabular}{|c|cc|cc|cc|}
\hline \multirow{2}{*}{$\operatorname{Re}$} & \multicolumn{2}{|c|}{ (Mittal, 2005) } & \multicolumn{2}{c|}{ (Qu et al., 2013) } & \multicolumn{2}{c|}{ Current } \\
& $\mathrm{Cd}$ & $\mathrm{St}$ & $\mathrm{Cd}$ & $\mathrm{St}$ & $\mathrm{Cd}$ & $\mathrm{St}$ \\
\hline 100 & 1.322 & 0.1644 & 1.317 & 0.1649 & 1.346 & 0.1648 \\
150 & $\sim \sim \sim$ & $\sim \sim \sim$ & 1.301 & 0.1841 & 1.329 & 0.1827 \\
200 & 1.327 & 0.1947 & 1.316 & 0.1958 & 1.336 & 0.1937 \\
\hline
\end{tabular}

Table 2: Summary and analysis of maximum force coefficient amplitude curves for impinging vortex of radius $\mathrm{r}_{\mathrm{c}}=1 \cdot \mathrm{D}$ impacting the cylinder at 25 TOI (Illustrated in Figure $3 \boldsymbol{b}$ ).

\begin{tabular}{|c|cccc|}
\hline \multirow{2}{*}{} & \multicolumn{4}{|c|}{ Maximum Force Coefficient Amplitudes } \\
\cline { 2 - 5 } & $\mathrm{Cl}(+)$ & $\mathrm{Cl}(-)$ & $\mathrm{Cd}(+)$ & $\mathrm{Cd}(-)$ \\
\hline Greatest & 1.851 & -2.952 & 1.128 & -0.735 \\
Least & 1.000 & -0.994 & 0.275 & -0.145
\end{tabular}




\begin{tabular}{|l|llll|} 
UP & $84.99 \%$ & $197.04 \%$ & $310.42 \%$ & $407.15 \%$ \\
\hline
\end{tabular}

*UP is under prediction.

Table 3: Mean and NSD for curves of maximum force coefficient amplitude for impinging vortex having radii of $1 \cdot \mathrm{D}, 2 \cdot \mathrm{D}$, and $3 \cdot \mathrm{D}$.

\begin{tabular}{|c|cccc|}
\hline \multirow{2}{*}{ Vortex } & \multicolumn{4}{|c|}{ Signed Maximum Amplitudes } \\
\cline { 2 - 5 } Radius & $\mathrm{Cl}(+)$ & $\mathrm{Cl}(-)$ & $\mathrm{Cd}(+)$ & $\mathrm{Cd}(-)$ \\
\hline $1 \cdot \mathrm{D}$ & 1.432 & -2.030 & 0.707 & -0.459 \\
$2 \cdot \mathrm{D}$ & 1.710 & -2.083 & 0.886 & -0.548 \\
$3 \cdot \mathrm{D}$ & 1.949 & -2.109 & 1.150 & -0.647 \\
\hline & \multicolumn{4}{|c}{ Mean-Normalized St. Deviation } \\
\hline & $20.34 \%$ & $33.44 \%$ & $42.75 \%$ & $45.90 \%$ \\
& $15.62 \%$ & $22.12 \%$ & $31.30 \%$ & $32.96 \%$ \\
& $11.09 \%$ & $6.91 \%$ & $17.86 \%$ & $20.91 \%$ \\
\hline
\end{tabular}

